\title{
Reduction of the planar double-box diagram for single-top production via auxiliary mass flow
}

\author{
Najam ul Basat, ${ }^{1,2 *}$ Zhao Li® ${ }^{1,2,3, \dagger}$ and Yefan Wang $\oplus^{1,2, *}$ \\ ${ }^{1}$ Institute of High Energy Physics, Chinese Academy of Sciences, Beijing 100049, China \\ ${ }^{2}$ School of Physics Sciences, University of Chinese Academy of Sciences, Beijing 100039, China \\ ${ }^{3}$ Center of High Energy Physics, Peking University, Beijing 100871, China
}

(Received 20 February 2021; accepted 13 August 2021; published 15 September 2021)

\begin{abstract}
The single-top production is an important process at the LHC to test the Standard Model and search for the new physics beyond the Standard Model. Although the complete next-to-next-to-leading order QCD correction to the single-top production is crucial, this calculation is still challenging at present. In order to efficiently reduce the next-to-next-to-leading order single-top amplitude, we improve the auxiliary mass flow method by introducing the $\epsilon$ truncation. For demonstration we choose one typical planar double-box diagram for the $t W$ production. It is shown that one coefficient of the form factors on its amplitude can be systematically reduced into the linear combination of 198 scalar integrals.
\end{abstract}

DOI: $10.1103 /$ PhysRevD.104.056020

\section{INTRODUCTION}

The top quark is the heaviest elementary particle in the Standard Model (SM). In 1994, the discovery of the top quark at the Tevatron $[1,2]$ meant the third generation of fermions in the SM is complete. Meanwhile the top quark is the only flavor that can decay before hadronization. This unique property provides an opportunity to directly measure the properties of the top quark. According to the fermion mass law [3], the top quark mass is related to the Yukawa strength between the top quark and the Higgs boson field. After the discovery of Higgs boson at the Large Hadron Collider (LHC), this coupling can be directly studied by the ATLAS and CMS collaborations [4-6]. Therefore, the top quark also plays an important role in the study on the electroweak symmetry breaking (EWSB).

At the hadron colliders, the dominant contribution to the top quark production is the top-pair production via the strong interaction, such as $q \bar{q} \rightarrow t \bar{t}$ and $g g \rightarrow t \bar{t}$. This process was discovered two decades ago [1,2]. Then the next largest contribution to the top quark production is the single-top production via the electroweak interaction, which was observed in 2009 at the Tevatron $[7,8]$ for the first time. Compared to the top-pair production, the $W t b$

\footnotetext{
*najam@ihep.ac.cn

tzhaoli@ihep.ac.cn

*wangyefan@ihep.ac.cn
}

Published by the American Physical Society under the terms of the Creative Commons Attribution 4.0 International license. Further distribution of this work must maintain attribution to the author(s) and the published article's title, journal citation, and DOI. Funded by SCOAP ${ }^{3}$. vertex is included in the single-top production, and can be directly used to measure the Cabibbo-Kobayashi-Maskawa (CKM) matrix element $\left|V_{t b}\right|$ without assuming unitarity $[9,10]$ and the extraction of the top quark mass $[11,12]$. On the other hand, the single-top production can be a sensitive probe to search for the new physics beyond the SM (BSM). For instance, the single-top production could be sensitive to the new heavy gauge boson $W^{\prime}$ [13-15], the new fermions [16-18] or the new scalars $[19,20]$. And it has been found that the single-top production provides a comfortable agreement with $2 \mathrm{HDM}+\alpha$ model [21-24] to search for the dark matter (DM).

At the LHC, there are three major modes for the single-top production: $s$-channel, $t$-channel, and the $t W$ production channel. The first two channels have been observed at the Tevatron [7,8]. And only recently the third channel, $t W$ production channel, was observed at the LHC [25]. Beside the progress of experiments, the precise theoretical predictions are demanded to match the high accuracy of experiment measurements. And the precise theoretical predictions can play vital roles in extracting important information from the experiment data. For all above three channels, the nextto-leading order QCD corrections have been investigated [26-37]. For the next-to-next-to-leading order (NNLO) corrections, many approximate results based on soft gluon resummation have been obtained [38-46]. The NNLO QCD corrections to the $t$ channel under structure function approximation have been calculated in [47]. Also the NNLO calculations including top-quark leptonic decay under structure function approximation and narrow width approximation have developed in recent years [48-51]. And the next-to-next-to-next-to-leading order soft-gluon corrections for the $t W$ production has been studied [52]. 
In the calculation of the multiloop Feynman diagrams, the amplitude generally needs to be reduced into linear combination of the master integrals, which can be further evaluated analytically or numerically. The first key step of amplitude reduction is the tensor reduction, which is used to separate the loop momenta from fermion chains or polarization vectors. The conventional approaches to the tensor reduction include the projection method [53-55] and the Tarasov's method [56]. However, in some multiscale processes, these approaches can be too complicated due to the difficulty of the inverse matrix or the dimension shift. Beside the conventional approaches, computational algebraic based algorithms [57-59] and numerical unitarity method [60-64] also have been developed in the last decade. Then, after the tensor reduction, the integration by part (IBP) identities are usually implemented to reduce the scalar integrals into the master integrals. In the past decade, many algorithms and codes have been developed for the IBP reduction [65-88].

Recently the auxiliary mass flow (AMF) method has been proposed to reduce the amplitude and the scalar integrals [89-91]. Also it can be used to numerically evaluate the master integrals and the phase space [92,93]. In amplitude reduction, this method can avoid complicated calculations of the inverse matrix and the dimension shift while the master integrals could be chosen freely.

In this paper, we introduce the truncation on $\epsilon$ to improve the efficiency of the matching procedure in the AMF method. With the help of this improvement, we can reduce one planar double-box diagram for the single-top production. Due to the complexity of multiloop multiscale diagram, we choose the integrals that include irreducible numerators to construct the set of scalar integrals. And in order to control the length of the reduction coefficients, we keep the reduction coefficients up to $\epsilon^{4}$, which is sufficient for the NNLO corrections. In the next section the main algorithm will be explained in detail. Then the reduction results will be shown. Finally the conclusion is made.

\section{AMPLITUDE REDUCTION VIA AUXILIARY MASS FLOW}

In general the loop amplitude can be written as

$$
\mathcal{M}=\int \mathbb{D}^{L} q \frac{N\left(\left\{q_{j}\right\}_{j=1}^{L},\left\{k_{e}\right\}_{e=1}^{E}\right)}{\prod_{i=1}^{n} \mathcal{D}_{i}^{\nu_{i}}}
$$

where $\mathbb{D}^{L} q \equiv \prod_{\ell=1}^{L}\left(\mathrm{~d}^{D} q_{\ell} /\left(\imath \pi^{D}\right)\right) .\left\{k_{e}\right\}_{e=1}^{E}$ are $E$ external momenta and $\left\{q_{j}\right\}_{j=1}^{L}$ are $L$ loop momenta. $\left\{\mathcal{D}_{i}\right\}_{i=1}^{n}$ are the denominators of propagators. $N\left(\left\{q_{j}\right\}_{j=1}^{L},\left\{k_{e}\right\}_{e=1}^{E}\right)$ is the numerator that may contain fermion chains or polarization vectors. In the AMF method, all the denominators are modified as [90]

$$
\frac{1}{\mathcal{D}_{i}} \equiv \frac{1}{P_{i}^{2}-m_{i}^{2}} \rightarrow \frac{1}{\tilde{\mathcal{D}}_{i}} \equiv \frac{1}{P_{i}^{2}-m_{i}^{2}+\imath \eta},
$$

where $\imath \eta$ is the auxiliary mass, $P_{i} \equiv Q_{i}+K_{i}$ is the momentum of the $i$ th propagator. $Q_{i}$ and $K_{i}$ are the linear combinations of, respectively, loop momenta and external momenta. Then we obtain the modified loop amplitude

$$
\widetilde{\mathcal{M}}(\eta)=\sum_{\substack{\mu_{1} \ldots \mu_{R} \\ \ell_{1} \ldots \ell_{R}}} N_{\mu_{1} \ldots \mu_{R}, \ell_{1} \ldots \ell_{R}}\left(\left\{k_{e}\right\}_{e=1}^{E}\right) \widetilde{G}_{\ell_{1} \ldots \ell_{R}}^{\mu_{1} \ldots \mu_{R}},
$$

where

$$
\tilde{G}_{\ell_{1} \ldots \ell_{R}}^{\mu_{1} \ldots \mu_{R}} \equiv \int \mathbb{D}^{L} q \frac{q_{\ell_{1}}^{\mu_{1}} \ldots q_{\ell_{R}}^{\mu_{R}}}{\prod_{i=1}^{n}\left[\left(Q_{i}+K_{i}\right)^{2}-m_{i}^{2}+\imath \eta\right]^{\nu_{i}}}
$$

is the modified tensor integral. And $N_{\mu_{1} \ldots \mu_{R}, \ell_{1} \ldots \ell_{R}}$ is the relevant coefficient. At the two-loop level we can define the modified amplitude explicitly

$$
\begin{aligned}
\tilde{\mathcal{M}}_{u v}(\eta) \equiv & \sum_{\mu_{1} \ldots \mu_{u+v}} N_{\mu_{1} \ldots \mu_{u+v}}, \underbrace{1, \ldots, 1}_{u} \underbrace{2, \ldots, 2}_{v}\left(\left\{k_{e}\right\}_{e=1}^{E}\right) \\
& \times \underbrace{\tilde{G}_{1}^{\mu_{1} \ldots \mu_{u+v}}}_{u} \underbrace{1, \ldots,}_{v} \underbrace{2, \ldots, 2},
\end{aligned}
$$

which only include one type of the tensor integrals. Then the two-loop modified amplitude can be written as

$$
\tilde{\mathcal{M}}(\eta)=\sum_{u, v} \tilde{\mathcal{M}}_{u v}(\eta)
$$

After the Feynman parametrization [94], by using Taylor series for $\eta \rightarrow \infty$ [90] we can obtain the series representation of $\tilde{\mathcal{M}}_{u v}(\eta)$

$$
\tilde{\mathcal{M}}_{u v}(\eta)=\sum_{i} \mathcal{C}_{i u v} \mathcal{F}_{i}
$$

where

$$
\begin{aligned}
\mathcal{C}_{\text {iuv }}= & \eta^{\operatorname{dim}\left(\mathcal{C}_{\text {iuv }}\right) / 2}\left(\sum_{p=0}^{p_{0}} \sum_{j} \sum_{\substack{\alpha_{1} \ldots \alpha_{t} \\
|\alpha|=p}}\left(a_{0 p \alpha j}(D) \eta^{-p} s^{\alpha} I_{2, j}^{(\mathrm{vac}), D}\right)\right. \\
& \left.+\mathcal{O}\left(\eta^{-p_{0}-1}\right)\right)
\end{aligned}
$$

Here $\mathcal{F}_{i}$ is the form factor and $\mathcal{C}_{i u v}$ is the relevant coefficient. $I_{2, j}^{(\mathrm{vac}), D}$ represents the $j$ th two-loop vacuum bubble master integral. $s \equiv\left(s_{1}, \ldots, s_{t}\right)$ is the tuple of linear independent kinematic variables $\left\{s_{1}, \ldots, s_{t}\right\} . s^{\alpha} \equiv s_{1}^{\alpha_{1}} \cdots s_{t}^{\alpha_{t}}$ is the monomial, where $\alpha=\left(\alpha_{1}, \ldots, \alpha_{t}\right)$ is a $t$ tuple of non-negative integers. And $a_{0 p \alpha j}$ is the coefficient that 
depends only on the space-time dimension $D$. The explicit definitions of symbols in Eq. (8) can be found in Ref. [90].

After obtaining the series representation of $\mathcal{C}_{\text {iuv }}$, we can choose a set of integrals for the reduction. The AMF method allows one to choose integrals freely. Thus in complex multiscale process we choose the integrals that include irreducible numerators to construct the set of modified scalar integrals. Here we define two-loop modified scalar integral

$\tilde{I} \equiv \int \mathbb{D}^{2} q \frac{\left(\prod_{e=1}^{E} \prod_{i=1}^{2}\left(k_{e} \cdot q_{i}\right)^{\rho_{e i}}\right)\left(\prod_{l=1}^{2} \prod_{j=1}^{l}\left(q_{j} \cdot q_{l}\right)^{\sigma_{j l}}\right)}{\prod_{i=1}^{n}\left[\left(Q_{i}+K_{i}\right)^{2}-m_{i}^{2}+\imath \eta\right]^{\nu_{i}}}$,

where the exponents $\rho_{e i}$ and $\sigma_{j l}$ are nonnegative integers. And we can define a tuple $\beta=\left(\beta_{1}, \beta_{2}\right)$, where

$$
\beta_{1} \equiv \sum_{e=1}^{E} \rho_{e, 1}+\sigma_{1,2}+2 \sigma_{1,1}
$$

and

$$
\beta_{2} \equiv \sum_{e=1}^{E} \rho_{e, 2}+\sigma_{1,2}+2 \sigma_{2,2} .
$$

In order to reduce $\tilde{\mathcal{M}}_{u v}(\eta)$, the set of modified scalar integrals can be chosen as

$$
\{\tilde{I}\}_{\beta=(u, v)} .
$$

Hence the set of modified scalar integrals has the same loop momenta rank and denominator powers with $\tilde{\mathcal{M}}_{u v}(\eta)$. We use $\tilde{I}_{u v k}$ to denote the $k$ th integral in $\{\tilde{I}\}_{\beta=(u, v)}$. For instance, since the number of independent external momenta is 3 , the reduction of $\tilde{\mathcal{M}}_{2,0}(\eta)$ needs seven modified scalar integrals,

$$
\begin{aligned}
& \tilde{I}_{2,0,1}(\eta) \equiv \int \mathbb{D}^{2} q \frac{\left(q_{1} \cdot q_{1}\right)}{\prod_{i=1}^{n}\left[\left(Q_{i}+K_{i}\right)^{2}-m_{i}^{2}+\imath \eta\right]^{\nu_{i}}}, \\
& \tilde{I}_{2,0,2}(\eta) \equiv \int \mathbb{D}^{2} q \frac{\left(k_{1} \cdot q_{1}\right)^{2}}{\prod_{i=1}^{n}\left[\left(Q_{i}+K_{i}\right)^{2}-m_{i}^{2}+\imath \eta\right]^{\nu_{i}}}, \\
& \tilde{I}_{2,0,3}(\eta) \equiv \int \mathbb{D}^{2} q \frac{\left(k_{1} \cdot q_{1}\right)\left(k_{2} \cdot q_{1}\right)}{\prod_{i=1}^{n}\left[\left(Q_{i}+K_{i}\right)^{2}-m_{i}^{2}+\imath \eta\right]^{\nu_{i}}}, \\
& \tilde{I}_{2,0,4}(\eta) \equiv \int \mathbb{D}^{2} q \frac{\left(k_{2} \cdot q_{1}\right)^{2}}{\prod_{i=1}^{n}\left[\left(Q_{i}+K_{i}\right)^{2}-m_{i}^{2}+\imath \eta\right]^{\nu_{i}}}, \\
& \tilde{I}_{2,0,5}(\eta) \equiv \int \mathbb{D}^{2} q \frac{\left(k_{1} \cdot q_{1}\right)\left(k_{3} \cdot q_{1}\right)}{\prod_{i=1}^{n}\left[\left(Q_{i}+K_{i}\right)^{2}-m_{i}^{2}+\imath \eta\right]^{\nu_{i}}}, \\
& \tilde{I}_{2,0,6}(\eta) \equiv \int \mathbb{D}^{2} q \frac{\left(k_{2} \cdot q_{1}\right)\left(k_{3} \cdot q_{1}\right)}{\prod_{i=1}^{n}\left[\left(Q_{i}+K_{i}\right)^{2}-m_{i}^{2}+\imath \eta\right]^{\nu_{i}}}, \\
& \tilde{I}_{2,0,7}(\eta) \equiv \int \mathbb{D}^{2} q \frac{\left(k_{3} \cdot q_{1}\right)^{2}}{\prod_{i=1}^{n}\left[\left(Q_{i}+K_{i}\right)^{2}-m_{i}^{2}+\imath \eta\right]^{\nu_{i}}} .
\end{aligned}
$$

Then by using Taylor series for $\eta \rightarrow \infty$ we can obtain the series representation of $\tilde{I}_{u v k}(\eta)$,

$$
\begin{aligned}
\tilde{I}_{u v k}= & \eta^{\operatorname{dim}\left(\tilde{I}_{u v k}\right) / 2}\left(\sum_{p=0}^{p_{0}} \sum_{j} \sum_{\substack{\alpha_{1} \ldots, \alpha_{t} \\
|\alpha|=p}}\left(a_{k p \alpha j}(D) \eta^{-p} S^{\alpha} I_{2, j}^{(\mathrm{vac}), D}\right)\right. \\
& \left.+\mathcal{O}\left(\eta^{-p_{0}-1}\right)\right)
\end{aligned}
$$

As an analog to the procedure in [90], by matching the form factor coefficient $\mathcal{C}_{i u v}$ and the set of modified scalar integrals $\left\{\tilde{I}_{u v k}\right\}$ in the series representation, one can generate the coefficient matrix $\mathbb{M}(D)$, where $D=4-2 \epsilon$ is the space-time dimension. Finally the reduction problem can be transformed into the null space problem of $\mathbb{M}(D)$,

$$
\mathbb{M}(D) \cdot \mathbb{X}(D)=0,
$$

where the coefficient matrix $\mathbb{M}(D)$ and the null space $\mathbb{X}(D)$ only depend on $D$. For the NNLO correction, the coefficient matrix usually can be large and complicated. Consequently the null space could be difficult to obtain, and the reduction coefficients can be very long.

To efficiently solve the null space and control the length of reduction coefficients in the dimension regularization, first we expand the coefficient matrix $\mathbb{M}(\epsilon)$ at $\epsilon \rightarrow 0$,

$$
\mathbb{M}(\epsilon)=\mathbb{M}_{0}+\mathbb{M}_{1} \epsilon+\cdots+\mathbb{M}_{m} \epsilon^{m}+\mathcal{O}\left(\epsilon^{m+1}\right),
$$

where $\mathbb{M}_{0}, \ldots, \mathbb{M}_{n}$ are constant matrices. Similarly the unknown null space $\mathbb{X}(\epsilon)$ can also be expanded as

$$
\mathbb{X}(\epsilon)=\mathbb{X}_{0}+\mathbb{X}_{1} \epsilon+\cdots+\mathbb{X}_{m} \epsilon^{m}+\mathcal{O}\left(\epsilon^{m+1}\right) .
$$

Then by substituting Eqs. (16) and (17) into Eq. (15) we can obtain a linear system of equations

$$
\begin{aligned}
& \mathbb{M}_{0} \cdot \mathbb{X}_{0}=0 \\
& \mathbb{M}_{0} \cdot \mathbb{X}_{1}+\mathbb{M}_{1} \cdot \mathbb{X}_{0}=0, \\
& \mathbb{M}_{0} \cdot \mathbb{X}_{2}+\mathbb{M}_{1} \cdot \mathbb{X}_{1}+\mathbb{M}_{2} \cdot \mathbb{X}_{0}=0, \\
& \quad \cdots \\
& \mathbb{M}_{0} \cdot \mathbb{X}_{m}+\mathbb{M}_{1} \cdot \mathbb{X}_{m-1}+\cdots+\mathbb{M}_{m} \cdot \mathbb{X}_{0}=0 .
\end{aligned}
$$

Starting from the $\epsilon^{0}$ order, we assume that the equation

$$
\mathbb{M}_{0} \cdot \mathbb{X}_{0}=0
$$

has $r_{0}$ solutions. Then we have

$$
\mathbb{M}_{0} \cdot \mathbb{X}_{0}^{\left(r_{0}\right)}=0
$$

where 


$$
\mathbb{X}_{0}^{\left(r_{0}\right)} \equiv\left(\mathbb{X}_{0}^{1}, \mathbb{X}_{0}^{2}, \ldots, \mathbb{X}_{0}^{r_{0}}\right)
$$

Since the linear combinations of $\left\{\mathbb{X}_{0}^{1}, \mathbb{X}_{0}^{2}, \ldots, \mathbb{X}_{0}^{r_{0}}\right\}$ are also the solutions of Eq. (20), the null space equation at $\epsilon^{1}$ order becomes

$$
\left(\mathbb{M}_{1} \cdot \mathbb{X}_{0}^{\left(r_{0}\right)}, \mathbb{M}_{0}\right)\left(\begin{array}{c}
\mathbb{C}_{0}^{\left(r_{0}, r_{1}\right)} \\
\mathbb{X}_{1}^{\left(r_{1}\right)}
\end{array}\right)=0
$$

where

$$
\mathbb{X}_{1}^{\left(r_{1}\right)} \equiv\left(\mathbb{X}_{1}^{1}, \mathbb{X}_{1}^{2}, \ldots, \mathbb{X}_{1}^{r_{1}}\right)
$$

are $r_{1}$ solutions, and $\mathbb{C}_{0}^{\left(r_{0}, r_{1}\right)}$ is $r_{0} \times r_{1}$ constant matrix. Now up to $\epsilon^{1}$, the solutions can be expressed as

$$
\mathbb{X}_{0}^{\left(r_{1}\right)}+\mathbb{X}_{1}^{\left(r_{1}\right)} \epsilon
$$

where $\mathbb{X}_{0}^{\left(r_{1}\right)} \equiv \mathbb{X}_{0}^{\left(r_{0}\right)} \cdot \mathbb{C}_{0}^{\left(r_{0}, r_{1}\right)}$.

Suppose that up to $\epsilon^{p}$ we have the solutions

$$
\mathbb{X}_{0}^{\left(r_{p}\right)}+\mathbb{X}_{1}^{\left(r_{p}\right)} \epsilon+\cdots+\mathbb{X}_{p}^{\left(r_{p}\right)} \epsilon^{p}
$$

Then at $\epsilon^{p+1}$ we can have

$$
\left(\mathbb{M}_{p+1} \cdot \mathbb{X}_{0}^{\left(r_{p}\right)}+\cdots+\mathbb{M}_{1} \cdot \mathbb{X}_{p}^{\left(r_{p}\right)}, \mathbb{M}_{0}\right) \cdot\left(\begin{array}{c}
\mathbb{C}_{p}^{\left(r_{p}, r_{p+1}\right)} \\
\mathbb{X}_{p+1}^{\left(r_{p+1}\right)}
\end{array}\right)=0
$$

Then up to the next order $\epsilon^{p+1}$ we can obtain the solution,

$$
\mathbb{X}_{0}^{\left(r_{p+1}\right)}+\mathbb{X}_{1}^{\left(r_{p+1}\right)} \epsilon+\cdots+\mathbb{X}_{p+1}^{\left(r_{p+1}\right)} \epsilon^{p+1},
$$

where

$$
\begin{aligned}
\mathbb{X}_{0}^{\left(r_{p+1}\right)} \equiv & \mathbb{X}_{0}^{\left(r_{p}\right)} \cdot \mathbb{C}_{p}^{\left(r_{p}, r_{p+1}\right)} \\
& \cdots \\
\mathbb{X}_{p}^{\left(r_{p}+1\right)} \equiv & \mathbb{X}_{p}^{\left(r_{p}\right)} \cdot \mathbb{C}_{p}^{\left(r_{p}, r_{p+1}\right)} .
\end{aligned}
$$

Therefore, by the iteration relations we can obtain the approximate solutions $\mathbb{X}^{\operatorname{approx}}(\epsilon)$,

$$
\mathbb{X}^{\operatorname{approx}}(\epsilon)=\mathbb{X}_{0}^{\left(r_{m}\right)}+\mathbb{X}_{1}^{\left(r_{m}\right)} \epsilon+\cdots+\mathbb{X}_{m}^{\left(r_{m}\right)} \epsilon^{m} .
$$

Since we take the truncation on $\epsilon$ in $\mathbb{M}(\epsilon)$ and $\mathbb{X}(\epsilon)$, the equations in Eq. (18) are the parts of the complete linear system of equations in Eq. (15). Meanwhile the approximation of the true solutions $\mathbb{X}(\epsilon)$ must exist within $\mathbb{X}^{\text {approx }}(\epsilon)$. And there could be some redundant solutions that satisfy Eq. (18) but not satisfy Eq. (15). To check if there are redundant solutions in $\mathbb{X}^{\text {approx }}(\epsilon)$, we can observe the number of the linear independent solutions in $\mathbb{X}^{\text {approx }}(\epsilon)$. If it is equal to the nullity of $\mathbb{M}(\epsilon)$, which can be obtained by randomly assigning $\epsilon$ as some constant numbers, it means that there is no redundant solution. Therefore, the $\mathbb{X}^{\text {approx }}(\epsilon)$ is the approximation of the $\mathbb{X}$,

$$
\mathbb{X}^{\text {approx }}(\epsilon)=\left.\mathbb{X}(\epsilon)\right|_{\epsilon^{m+1}=0} .
$$

If there are redundant solutions in $\mathbb{X}^{\text {approx }}(\epsilon)$, we can use higher $\epsilon$ order expansions to introduce more constraints to the linear system in Eq. (18). Then we repeat the above procedure until there are no redundant solutions in $\mathbb{X}^{\text {approx }}(\epsilon)$.

The finite fields are implemented to improve the efficiency in null space calculations of the constant matrices, and the Chinese remainder theorem is used to reconstruct the rational numbers. Finally we keep the reduction coefficients up to $\epsilon^{4}$ since at the two-loop level the maximum divergence of integrals is $\epsilon^{-4}$. Consequently the null space problem can be efficiently solved. And the length of reduction coefficients can be effectively controlled.

After the reduction, $\tilde{\mathcal{M}}_{u v}(\eta)$ can be reduced to several modified scalar integrals

$$
\tilde{\mathcal{M}}_{u v}(\eta)=\sum_{i} \sum_{k} C_{i u v k} \tilde{I}_{u v k}(\eta) \mathcal{F}_{i}
$$

where $C_{i u v k}$ is the reduction coefficient of relevant $\tilde{I}_{u v k}(\eta)$ and $\mathcal{F}_{i}$ for $\tilde{\mathcal{M}}_{u v}(\eta)$. Since the set of modified scalar integrals $\{\tilde{I}\}_{\beta=(u, v)}$ and $\tilde{\mathcal{M}}_{u v}(\eta)$ have same denominator powers and loop momenta rank, the reduction coefficients $\left\{C_{i u v k}\right\}$ only depend on the numerators of amplitude and modified scalar integrals. And the auxiliary mass $i \eta$ only exist in the denominators of amplitude and scalar integrals. Consequently, the reduction coefficients $\left\{C_{i u v k}\right\}$ are independent of $\eta$.

For given form factor $\mathcal{F}_{i}$ of amplitude, the set $\left\{\tilde{I}_{u v k}\right\}$ and $\left\{C_{i u v k}\right\}$ can be ordered using certain well order relation, e.g., lexicographical ordering, for $(u, v, k)$, respectively. And $\tilde{I}_{p}$ and $C_{i p}$ can be denoted as the $p$ th element in the corresponding set. Finally the modified amplitude can be written as

$$
\tilde{\mathcal{M}}(\eta)=\sum_{u, v} \tilde{\mathcal{M}}_{u v}(\eta)=\sum_{i} \sum_{p} C_{i p} \tilde{I}_{p}(\eta) \mathcal{F}_{i} .
$$

Since the $C_{i p}$ is independent of $\eta$, When $\eta \rightarrow 0$, the original amplitude can be written as

$$
\mathcal{M}=\sum_{i} \sum_{p} C_{i p} I_{p} \mathcal{F}_{i}
$$

These scalar integrals $\left\{I_{p}\right\}$ can be further reduced into final master integrals via auxiliary mass flow or other methods. 


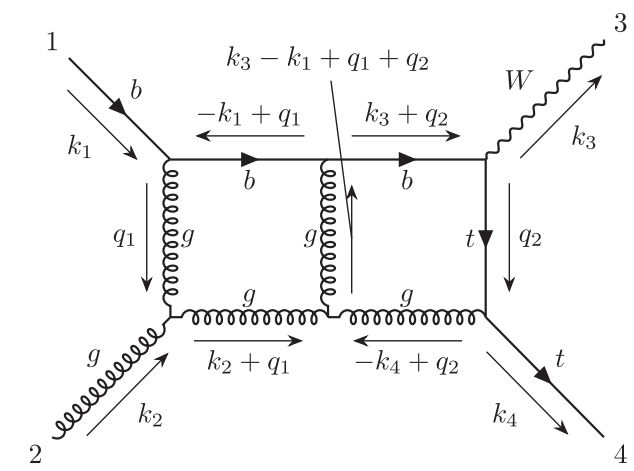

FIG. 1. Double-box diagram for the $t W$ production.

\section{PLANAR DOUBLE-BOX DIAGRAM IN THE SINGLE-TOP PRODUCTION}

In this section, we implement our improved approach on one double-box diagram of the $t W$ production process $b\left(k_{1}\right)+g\left(k_{2}\right) \rightarrow W\left(k_{3}\right)+t\left(k_{4}\right)$. The Feynman diagram is shown in Fig. 1, which is plotted by LaTeX package TikZ-Feynman [95].

Its relevant modified amplitude can be written as

$$
\tilde{\mathcal{M}}(\eta)=\int \mathbb{D}^{2} q \frac{N\left(q_{1}, q_{2}, k_{1}, k_{2}, k_{3}, k_{4}\right)}{\tilde{\mathcal{D}}_{1} \tilde{\mathcal{D}}_{2} \tilde{\mathcal{D}}_{3} \tilde{\mathcal{D}}_{4} \tilde{\mathcal{D}}_{5} \tilde{\mathcal{D}}_{6} \tilde{\mathcal{D}}_{7}}
$$

where the denominators are

$$
\begin{aligned}
& \tilde{\mathcal{D}}_{1}=\left(q_{1}-k_{1}\right)^{2}+\imath \eta, \\
& \tilde{\mathcal{D}}_{2}=\left(q_{1}+k_{2}\right)^{2}+\imath \eta, \\
& \tilde{\mathcal{D}}_{3}=\left(k_{3}-k_{1}+q_{1}+q_{2}\right)^{2}+\imath \eta, \\
& \tilde{\mathcal{D}}_{4}=\left(k_{3}+q_{2}\right)^{2}+\imath \eta, \\
& \tilde{\mathcal{D}}_{5}=\left(q_{2}-k_{4}\right)^{2}+\imath \eta, \\
& \tilde{\mathcal{D}}_{6}=q_{1}^{2}+\imath \eta, \\
& \tilde{\mathcal{D}}_{7}=q_{2}^{2}-m_{t}^{2}+\imath \eta .
\end{aligned}
$$

Here $m_{W}$ is the mass of $W$ boson and $m_{t}$ is the mass of top quark. And we define

$$
\begin{aligned}
& s_{1} \equiv 2\left(k_{1} \cdot k_{2}\right), \\
& s_{2} \equiv m_{W}^{2}-2\left(k_{1} \cdot k_{3}\right) .
\end{aligned}
$$

For reader's convenience we explicitly show the numerator of this amplitude

$$
\begin{aligned}
N\left(q_{1}, q_{2}, k_{1}, k_{2}, k_{3}, k_{4}\right)= & -l g_{49} g_{10}^{2} g_{11}^{3} \varepsilon^{\mu_{5}}\left(k_{2}\right) \bar{u}\left(k_{4}\right) \gamma^{\mu_{1}}\left(\not 12+m_{t}\right) \not \psi^{\prime}\left(k_{3}\right) P_{L}(\not 12+\not k 3) \gamma^{\mu_{2}}(\not k 1-\not 1) \gamma^{\mu_{3}} u\left(k_{1}\right) \\
& \times\left\{\left(q_{1}-k_{2}\right)^{\mu_{4}} g^{\mu_{3} \mu_{5}}+\left(2 k_{2}+q_{1}\right)^{\mu_{3}} g^{\mu_{4} \mu_{5}}-\left(k_{2}+2 q_{1}\right)^{\mu_{5}} g^{\mu_{3} \mu_{4}}\right\} \\
& \times\left\{\left(2 k_{4}-k_{2}-q_{1}+2 q_{2}\right)^{\mu_{4}} g^{\mu_{1} \mu_{2}}+\left(2 q_{1}+q_{2}+2 k_{2}-k_{4}\right)^{\mu_{1}} g^{\mu_{2} \mu_{4}}+\left(-q_{1}+q_{2}-k_{2}-k_{4}\right)^{\mu_{2}} g^{\mu_{1} \mu_{4}}\right\} .
\end{aligned}
$$

From this amplitude we can extract 10 linear independent form factors:

$$
\begin{aligned}
& \mathcal{F}_{1}=\bar{u}\left(k_{4}\right) P_{L} \phi\left(k_{2}\right) \phi^{*}\left(k_{3}\right) u\left(k_{1}\right), \\
& \mathcal{F}_{2}=\bar{u}\left(k_{4}\right) P_{L} \phi\left(k_{2}\right) \not k_{2} u\left(k_{1}\right)\left(k_{2} \cdot \varepsilon^{*}\left(k_{3}\right)\right), \\
& \mathcal{F}_{3}=\bar{u}\left(k_{4}\right) P_{L} \phi^{*}\left(k_{3}\right) \not_{2} u\left(k_{1}\right)\left(k_{3} \cdot \varepsilon\left(k_{2}\right)\right), \\
& \mathcal{F}_{4}=\bar{u}\left(k_{4}\right) P_{L} u\left(k_{1}\right)\left(k_{3} \cdot \varepsilon\left(k_{2}\right)\right)\left(k_{2} \cdot \varepsilon^{*}\left(k_{3}\right)\right), \\
& \mathcal{F}_{5}=\bar{u}\left(k_{4}\right) P_{L} u\left(k_{1}\right)\left(\varepsilon\left(k_{2}\right) \cdot \varepsilon^{*}\left(k_{3}\right)\right), \\
& \mathcal{F}_{6}=\bar{u}\left(k_{4}\right) P_{R} \phi\left(k_{2}\right) \phi^{*}\left(k_{3}\right) \not k_{2} u\left(k_{1}\right), \\
& \mathcal{F}_{7}=\bar{u}\left(k_{4}\right) P_{R} \phi\left(k_{2}\right) u\left(k_{1}\right)\left(k_{2} \cdot \varepsilon^{*}\left(k_{3}\right)\right), \\
& \mathcal{F}_{8}=\bar{u}\left(k_{4}\right) P_{R} \phi^{*}\left(k_{3}\right) u\left(k_{1}\right)\left(k_{3} \cdot \varepsilon\left(k_{2}\right)\right), \\
& \mathcal{F}_{9}=\bar{u}\left(k_{4}\right) P_{R} \not k_{2} u\left(k_{1}\right)\left(k_{2} \cdot \varepsilon^{*}\left(k_{3}\right)\right)\left(k_{3} \cdot \varepsilon\left(k_{2}\right)\right), \\
& \mathcal{F}_{10}=\bar{u}\left(k_{4}\right) P_{R} \not k_{2} u\left(k_{1}\right)\left(\varepsilon\left(k_{2}\right) \cdot \varepsilon^{*}\left(k_{3}\right)\right) .
\end{aligned}
$$

In projection method, to finish the tensor reduction of the amplitude, one needs to calculate the analytical inversion of the projection matrix, which includes four independent kinematic variables and space-time dimension $D$. Since the symbolic matrix is big and complex, the analytical calculation of its inversion is quite difficult.

As mentioned in last section, the modified amplitude can be decomposed into 15 parts

$$
\begin{aligned}
\tilde{\mathcal{M}}(\eta)= & \tilde{\mathcal{M}}_{0,0}(\eta)+\tilde{\mathcal{M}}_{0,1}(\eta)+\tilde{\mathcal{M}}_{1,0}(\eta)+\tilde{\mathcal{M}}_{0,2}(\eta) \\
& +\tilde{\mathcal{M}}_{1,1}(\eta)+\tilde{\mathcal{M}}_{2,0}(\eta)+\tilde{\mathcal{M}}_{0,3}(\eta)+\tilde{\mathcal{M}}_{1,2}(\eta) \\
& +\tilde{\mathcal{M}}_{2,1}(\eta)+\tilde{\mathcal{M}}_{3,0}(\eta)+\tilde{\mathcal{M}}_{1,3}(\eta)+\tilde{\mathcal{M}}_{2,2}(\eta) \\
& +\tilde{\mathcal{M}}_{3,1}(\eta)+\tilde{\mathcal{M}}_{2,3}(\eta)+\tilde{\mathcal{M}}_{3,2}(\eta) .
\end{aligned}
$$

In this paper we show the reduction results for the coefficient of $\mathcal{F}_{1}$. The reductions of the other form factors can be finished in the same way. And the reduction difficulty of the other form factors is in the same level of $\mathcal{F}_{1}$. For simplicity, we only show the scalar integrals $I_{p}$ with nonzero reduction coefficients. Consequently 198 out of 486 scalar integrals are remaining. The reduction coefficients are kept up to $\epsilon^{4}$. And the length of reduction coefficients can be effectively controlled. For convenience 
the constant factor $l g_{49} g_{10}^{2} g_{11}^{3}$ is factorized out in the results. For instance,

$$
I_{198} \equiv \int \mathbb{D}^{2} q \frac{\left(k_{3} \cdot q_{1}\right)\left(q_{1} \cdot q_{1}\right)\left(q_{2} \cdot q_{2}\right)}{\mathcal{D}_{1} \mathcal{D}_{2} \mathcal{D}_{3} \mathcal{D}_{4} \mathcal{D}_{5} \mathcal{D}_{6} \mathcal{D}_{7}}
$$

By using the in-house package SeRA.jl, the corresponding reduction coefficient ${ }^{1}$ is

$$
C_{1,198}=\frac{2 s_{1} m_{t}\left(1+5 \epsilon+12 \epsilon^{2}+24 \epsilon^{3}+48 \epsilon^{4}\right)}{m_{t}^{2}\left(m_{W}^{2}-s_{2}\right)+s_{2}\left(-m_{W}^{2}+s_{1}+s_{2}\right)} .
$$

To cross check the coefficients $\left\{C_{1, p}\right\}$, we use the Tarasov's method [56] and IBP reduction to reduce the original amplitude numerically. Then we also apply the numerical IBP reduction to $\left\{I_{p}\right\}$. Finally the two reduction results are consistent. In IBP reduction procedure we use packages FIRE [68,69] and LiteRed [75].

\footnotetext{
${ }^{1}$ The complete expressions of the set of scalar integrals and their reduction coefficients can be downloaded at https://github .com/zhaoli-IHEP/gbtw_Reduction_Data.
}

\section{CONCLUSION}

In this paper, we improve the AMF method by taking the truncation on $\epsilon$ in the matching procedure. And we reduce one planar double-box diagram for the $t W$ production as the demonstration. The amplitude can be easily reduced into 10 form factors. One coefficient of the form factors can be easily reduced into 198 scalar integrals which include irreducible numerators. And the length of the reduction coefficients can be effectively controlled. This approach can be implemented on some other important processes in the future, such as the other diagrams in the NNLO $t W$ production.

\section{ACKNOWLEDGMENTS}

This work was supported by the National Natural Science Foundation of China under Grants No. 11675185 and No. 12075251. N. B. would like to acknowledge financial support from CAS-TWAS President's Fellowship Program 2017. The authors want to thank Yan-Qing Ma, Jian Wang, and Yang Zhang for helpful discussions.
[1] S. Abachi et al. (D0 Collaboration), Observation of the Top Quark, Phys. Rev. Lett. 74, 2632 (1995).

[2] F. Abe et al. (CDF Collaboration), Observation of Top Quark Production in $\bar{p} p$ Collisions, Phys. Rev. Lett. 74, 2626 (1995).

[3] S. Weinberg, A Model of Leptons, Phys. Rev. Lett. 19, 1264 (1967).

[4] G. Aad et al. (ATLAS Collaboration), Evidence for single top-quark production in the $s$-channel in proton-proton collisions at $\sqrt{s}=8 \mathrm{TeV}$ with the ATLAS detector using the matrix element method, Phys. Lett. B 756, 228 (2016).

[5] G. Aad et al. (ATLAS Collaboration), Measurement of the production cross-section of a single top quark in association with a $W$ boson at $8 \mathrm{TeV}$ with the ATLAS experiment, J. High Energy Phys. 01 (2016) 064.

[6] G. Aad et al. (ATLAS Collaboration), Search for anomalous couplings in the $W t b$ vertex from the measurement of double differential angular decay rates of single top quarks produced in the $t$-channel with the ATLAS detector, J. High Energy Phys. 04 (2016) 023.

[7] T. Aaltonen et al. (CDF Collaboration), First Observation of Electroweak Single Top Quark Production, Phys. Rev. Lett. 103, 092002 (2009).

[8] V. Abazov et al. (D0 Collaboration), Observation of Single Top Quark Production, Phys. Rev. Lett. 103, 092001 (2009).

[9] J. Alwall, R. Frederix, J.-M. Gerard, A. Giammanco, M. Herquet, S. Kalinin, E. Kou, V. Lemaitre, and F. Maltoni, Is $\mathrm{V}\left({ }_{t b}\right) \simeq 1$ ?, Eur. Phys. J. C 49, 791 (2007).
[10] Q.-H. Cao and B. Yan, Determining $V_{t b}$ at electron-positron colliders, Phys. Rev. D 92, 094018 (2015).

[11] A. M. Sirunyan et al. (CMS Collaboration), Measurement of the top quark mass using single top quark events in protonproton collisions at $\sqrt{s}=8 \mathrm{TeV}$, Eur. Phys. J. C 77, 354 (2017).

[12] S. Alekhin, S. Moch, and S. Thier, Determination of the topquark mass from hadro-production of single top-quarks, Phys. Lett. B 763, 341 (2016).

[13] E. Malkawi, T. M. Tait, and C. Yuan, A model of strong flavor dynamics for the top quark, Phys. Lett. B 385, 304 (1996).

[14] K. Hsieh, K. Schmitz, J.-H. Yu, and C.-P. Yuan, Global analysis of general $\mathrm{SU}(2) \times \mathrm{SU}(2) \times \mathrm{U}(1)$ models with precision data, Phys. Rev. D 82, 035011 (2010).

[15] Q.-H. Cao, Z. Li, J.-H. Yu, and C. Yuan, Discovery and identification of $\mathrm{W}^{\prime}$ and $\mathrm{Z}^{\prime}$ in $\mathrm{SU}(2) \times \mathrm{SU}(2) \times$ U(1) models at the LHC, Phys. Rev. D 86, 095010 (2012).

[16] Q.-H. Cao, C. S. Li, and C.-P. Yuan, Impact of single-top measurement to littlest Higgs model with T-parity, Phys. Lett. B 668, 24 (2008).

[17] E. L. Berger, Q.-H. Cao, C.-R. Chen, and H. Zhang, Top quark polarization as a probe of models with extra gauge bosons, Phys. Rev. D 83, 114026 (2011).

[18] E. L. Berger, Q.-H. Cao, J.-H. Yu, and C.-P. Yuan, Calculation of associated production of a top quark and a W' at the LHC, Phys. Rev. D 84, 095026 (2011). 
[19] E. Drueke, J. Nutter, R. Schwienhorst, N. Vignaroli, D. G. E. Walker, and J.-H. Yu, Single top production as a probe of heavy resonances, Phys. Rev. D 91, 054020 (2015).

[20] Q.-H. Cao, X. Wan, X.-P. Wang, and S.-h. Zhu, Searching for charged Higgs boson in polarized top quark, Phys. Rev. D 87, 055022 (2013).

[21] P. Pani and G. Polesello, Dark matter production in association with a single top-quark at the LHC in a twoHiggs-doublet model with a pseudoscalar mediator, Phys. Dark Universe 21, 8 (2018).

[22] M. Bauer, U. Haisch, and F. Kahlhoefer, Simplified dark matter models with two Higgs doublets: I. Pseudoscalar mediators, J. High Energy Phys. 05 (2017) 138.

[23] T. Abe et al. (LHC Dark Matter Working Group Collaboration), LHC Dark Matter Working Group: Next-generation spin-0 dark matter models, Phys. Dark Universe 27, 100351 (2020).

[24] G. Aad et al. (ATLAS Collaboration), Search for dark matter produced in association with a single top quark in $\sqrt{s}=13 \mathrm{TeV} p p$ collisions with the ATLAS detector, arXiv:2011.09308.

[25] S. Chatrchyan et al. (CMS Collaboration), Observation of the Associated Production of a Single Top Quark and a $W$ Boson in $p p$ Collisions at $\sqrt{s}=8 \mathrm{TeV}$, Phys. Rev. Lett. 112, 231802 (2014).

[26] G. Bordes and B. van Eijk, Calculating QCD corrections to single top production in hadronic interactions, Nucl. Phys. B435, 23 (1995).

[27] M. C. Smith and S. Willenbrock, QCD and Yukawa corrections to single top quark production via $q \bar{q} \rightarrow t \bar{b}$, Phys. Rev. D 54, 6696 (1996).

[28] S. Zhu, Next-to-leading order QCD corrections to $b g \rightarrow t W^{-}$at CERN large hadron collider, Phys. Lett. B 524, 283 (2002); 537, 351(E) (2002).

[29] B. Harris, E. Laenen, L. Phaf, Z. Sullivan, and S. Weinzierl, The fully differential single top quark cross-section in next to leading order QCD, Phys. Rev. D 66, 054024 (2002).

[30] Z. Sullivan, Understanding single-top-quark production and jets at hadron colliders, Phys. Rev. D 70, 114012 (2004).

[31] J. M. Campbell, R. Ellis, and F. Tramontano, Single top production and decay at next-to-leading order, Phys. Rev. D 70, 094012 (2004).

[32] Q.-H. Cao and C.-P. Yuan, Single top quark production and decay at next-to-leading order in hadron collision, Phys. Rev. D 71, 054022 (2005).

[33] Q.-H. Cao, R. Schwienhorst, and C.-P. Yuan, Next-toleading order corrections to single top quark production and decay at Tevatron. 1. $s^{-}$channel process, Phys. Rev. D 71, 054023 (2005).

[34] Q.-H. Cao, R. Schwienhorst, J. A. Benitez, R. Brock, and C.-P. Yuan, Next-to-leading order corrections to single top quark production and decay at the Tevatron: 2. $t^{-}$channel process, Phys. Rev. D 72, 094027 (2005).

[35] Q.-H. Cao, Demonstration of one cutoff phase space slicing method: Next-to-leading order QCD corrections to the tW associated production in hadron collision, arXiv:0801.1539.

[36] J. M. Campbell, R. Frederix, F. Maltoni, and F. Tramontano, NLO predictions for t-channel production of single top and fourth generation quarks at hadron colliders, J. High Energy Phys. 10 (2009) 042.
[37] S. Heim, Q.-H. Cao, R. Schwienhorst, and C.-P. Yuan, Nextto-leading order QCD corrections to s-channel single top quark production and decay at the LHC, Phys. Rev. D 81, 034005 (2010).

[38] S. Mrenna and C. P. Yuan, Effects of QCD resummation on $\mathrm{W}+\mathrm{h}$ and $\mathrm{t}$ anti-b production at the Tevatron, Phys. Lett. B 416, 200 (1998).

[39] N. Kidonakis, Single top production at the Tevatron: Threshold resummation and finite-order soft gluon corrections, Phys. Rev. D 74, 114012 (2006).

[40] N. Kidonakis, Higher-order soft gluon corrections in single top quark production at the LHC, Phys. Rev. D 75, 071501 (2007).

[41] N. Kidonakis, Two-loop soft anomalous dimensions for single top quark associated production with a W- or $\mathrm{H}$-, Phys. Rev. D 82, 054018 (2010).

[42] N. Kidonakis, Next-to-next-to-leading soft-gluon corrections for the top quark cross section and transverse momentum distribution, Phys. Rev. D 82, 114030 (2010).

[43] N. Kidonakis, Next-to-next-to-leading-order collinear and soft gluon corrections for t-channel single top quark production, Phys. Rev. D 83, 091503 (2011).

[44] Q.-H. Cao, P. Sun, B. Yan, C. P. Yuan, and F. Yuan, Transverse momentum resummation for $t$-channel single top quark production at the LHC, Phys. Rev. D 98, 054032 (2018).

[45] P. Sun, B. Yan, and C. P. Yuan, Transverse momentum resummation for $s$-channel single top quark production at the LHC, Phys. Rev. D 99, 034008 (2019).

[46] C. S. Li, H. T. Li, D. Y. Shao, and J. Wang, Momentumspace threshold resummation in $t W$ production at the LHC, J. High Energy Phys. 06 (2019) 125.

[47] M. Brucherseifer, F. Caola, and K. Melnikov, On the NNLO QCD corrections to single-top production at the LHC, Phys. Lett. B 736, 58 (2014).

[48] E. L. Berger, J. Gao, C. P. Yuan, and H. X. Zhu, NNLO QCD corrections to t-channel single top-quark production and decay, Phys. Rev. D 94, 071501 (2016).

[49] E. L. Berger, J. Gao, and H. X. Zhu, Differential distributions for t-channel single top-quark production and decay at next-to-next-to-leading order in QCD, J. High Energy Phys. 11 (2017) 158.

[50] Z. L. Liu and J. Gao, s -channel single top quark production and decay at next-to-next-to-leading-order in QCD, Phys. Rev. D 98, 071501 (2018).

[51] J. Campbell, T. Neumann, and Z. Sullivan, Single-top-quark production in the $t$-channel at NNLO, J. High Energy Phys. 02 (2021) 040.

[52] N. Kidonakis, Soft-gluon corrections for $t W$ production at $\mathrm{N}^{3}$ LO, Phys. Rev. D 96, 034014 (2017).

[53] T. Binoth, E. W. N. Glover, P. Marquard, and J. J. van der Bij, Two loop corrections to light by light scattering in supersymmetric QED, J. High Energy Phys. 05 (2002) 060.

[54] E. W. N. Glover, Two loop QCD helicity amplitudes for massless quark quark scattering, J. High Energy Phys. 04 (2004) 021.

[55] Y. Wang and Z. Li, Extended projection method for massive fermions, Chin. Phys. C 44, 033102 (2020).

[56] O. V. Tarasov, Connection between Feynman integrals having different values of the space-time dimension, Phys. Rev. D 54, 6479 (1996). 
[57] P. Mastrolia and G. Ossola, On the integrand-reduction method for two-loop scattering amplitudes, J. High Energy Phys. 11 (2011) 014.

[58] S. Badger, H. Frellesvig, and Y. Zhang, Hepta-cuts of twoloop scattering amplitudes, J. High Energy Phys. 04 (2012) 055.

[59] Y. Zhang, Integrand-level reduction of loop amplitudes by computational algebraic geometry methods, J. High Energy Phys. 09 (2012) 042.

[60] S. Abreu, F. F. Cordero, H. Ita, M. Jaquier, B. Page, and M. Zeng, Two-Loop Four-Gluon Amplitudes from Numerical Unitarity, Phys. Rev. Lett. 119, 142001 (2017).

[61] S. Abreu, F. F. Cordero, H. Ita, B. Page, and M. Zeng, Planar two-loop five-gluon amplitudes from numerical unitarity, Phys. Rev. D 97, 116014 (2018).

[62] S. Badger, C. Bronnum-Hansen, H. B. Hartanto, and T. Peraro, First Look at Two-Loop Five-Gluon Scattering in QCD, Phys. Rev. Lett. 120, 092001 (2018).

[63] S. Abreu, J. Dormans, F. Febres Cordero, H. Ita, and B. Page, Analytic Form of Planar Two-Loop Five-Gluon Scattering Amplitudes in QCD, Phys. Rev. Lett. 122, 082002 (2019).

[64] S. Abreu, F. Febres Cordero, H. Ita, B. Page, and V. Sotnikov, Planar two-loop five-parton amplitudes from numerical unitarity, J. High Energy Phys. 11 (2018) 116.

[65] S. Laporta, High precision calculation of multiloop Feynman integrals by difference equations, Int. J. Mod. Phys. A 15, 5087 (2000).

[66] C. Anastasiou and A. Lazopoulos, Automatic integral reduction for higher order perturbative calculations, J. High Energy Phys. 07 (2004) 046.

[67] A. V. Smirnov, Algorithm FIRE-Feynman integral reduction, J. High Energy Phys. 10 (2008) 107.

[68] A. V. Smirnov, FIRE5: a C++ implementation of Feynman integral reduction, Comput. Phys. Commun. 189, 182 (2015).

[69] A. V. Smirnov and F. S. Chuharev, FIRE6: Feynman integral reduction with modular arithmetic, Comput. Phys. Commun. 247, 106877 (2020).

[70] P. Maierhfer, J. Usovitsch, and P. Uwer, Kiraa Feynman integral reduction program, Comput. Phys. Commun. 230, 99 (2018).

[71] P. Maierhfer and J. Usovitsch, Kira 1.2 Release notes, arXiv:1812.01491.

[72] C. Studerus, Reduze-Feynman integral reduction in $\mathrm{C}++$, Comput. Phys. Commun. 181, 1293 (2010).

[73] A. von Manteuffel and C. Studerus, Reduze 2-Distributed Feynman integral reduction, arXiv:1201.4330.

[74] R. N. Lee, Presenting litered: A tool for the loop integrals reduction, arXiv:1212.2685.

[75] R. N. Lee, LiteRed 1.4: A powerful tool for reduction of multiloop integrals, J. Phys. Conf. Ser. 523, 012059 (2014).

[76] A. Georgoudis, K. J. Larsen, and Y. Zhang, Cristal and Azurite: new tools for integration-by-parts reductions, Proc. Sci., RADCOR2017 (2017) 020 [arXiv:1712.07510].
[77] D. Bendle, J. Boehm, W. Decker, A. Georgoudis, F.-J. Pfreundt, M. Rahn, P. Wasser, and Y. Zhang, Integration-byparts reductions of Feynman integrals using singular and GPI-space, J. High Energy Phys. 02 (2020) 079.

[78] A. V. Smirnov, An Algorithm to construct Grobner bases for solving integration by parts relations, J. High Energy Phys. 04 (2006) 026.

[79] R. N. Lee, Group structure of the integration-by-part identities and its application to the reduction of multiloop integrals, J. High Energy Phys. 07 (2008) 031.

[80] R. M. Schabinger, A new algorithm for the generation of unitarity-compatible integration by parts relations, J. High Energy Phys. 01 (2012) 077.

[81] K. J. Larsen and Y. Zhang, Integration-by-parts reductions from unitarity cuts and algebraic geometry, Phys. Rev. D 93, 041701 (2016).

[82] J. Bhm, A. Georgoudis, K. J. Larsen, M. Schulze, and Y. Zhang, Complete sets of logarithmic vector fields for integration-by-parts identities of Feynman integrals, Phys. Rev. D 98, 025023 (2018).

[83] A. von Manteuffel and R. M. Schabinger, A novel approach to integration by parts reduction, Phys. Lett. B 744, 101 (2015).

[84] D. A. Kosower, Direct solution of integration-by-parts systems, Phys. Rev. D 98, 025008 (2018).

[85] J. Böhm, A. Georgoudis, K. J. Larsen, H. Schönemann, and Y. Zhang, Complete integration-by-parts reductions of the non-planar hexagon-box via module intersections, J. High Energy Phys. 09 (2018) 024.

[86] H. A. Chawdhry, M. A. Lim, and A. Mitov, Two-loop fivepoint massless QCD amplitudes within the integration-byparts approach, Phys. Rev. D 99, 076011 (2019).

[87] P. Mastrolia and S. Mizera, Feynman integrals and intersection theory, J. High Energy Phys. 02 (2019) 139.

[88] J. Boehm, M. Wittmann, Z. Wu, Y. Xu, and Y. Zhang, IBP reduction coefficients made simple, J. High Energy Phys. 12 (2020) 054.

[89] X. Liu and Y.-Q. Ma, Determining arbitrary Feynman integrals by vacuum integrals, Phys. Rev. D 99, 071501 (2019).

[90] Y. Wang, Z. Li, and N. U1 Basat, Direct reduction of multiloop multiscale scattering amplitudes, Phys. Rev. D 101, 076023 (2020).

[91] X. Guan, X. Liu, and Y.-Q. Ma, Complete reduction of integrals in two-loop five-light-parton scattering amplitudes, Chin. Phys. C 44, 093106 (2020).

[92] X. Liu, Y.-Q. Ma, and C.-Y. Wang, A systematic and efficient method to compute multi-loop master integrals, Phys. Lett. B 779, 353 (2018).

[93] X. Liu, Y.-Q. Ma, W. Tao, and P. Zhang, Calculation of Feynman loop integration and phase-space integration via auxiliary mass flow, Chin. Phys. C 45, 013115 (2021).

[94] G. Heinrich, Sector decomposition, Int. J. Mod. Phys. A 23, 1457 (2008).

[95] J. Ellis, TikZ-Feynman: Feynman diagrams with TikZ, Comput. Phys. Commun. 210, 103 (2017). 